\title{
THERMOLUMINESCENCE INDUCED BY GAMMA RADIATION IN LiCsSO4 FERROELASTIC CRYSTAL DOPED WITH DIFFERENT HEAVY METALS
}

\author{
By
}

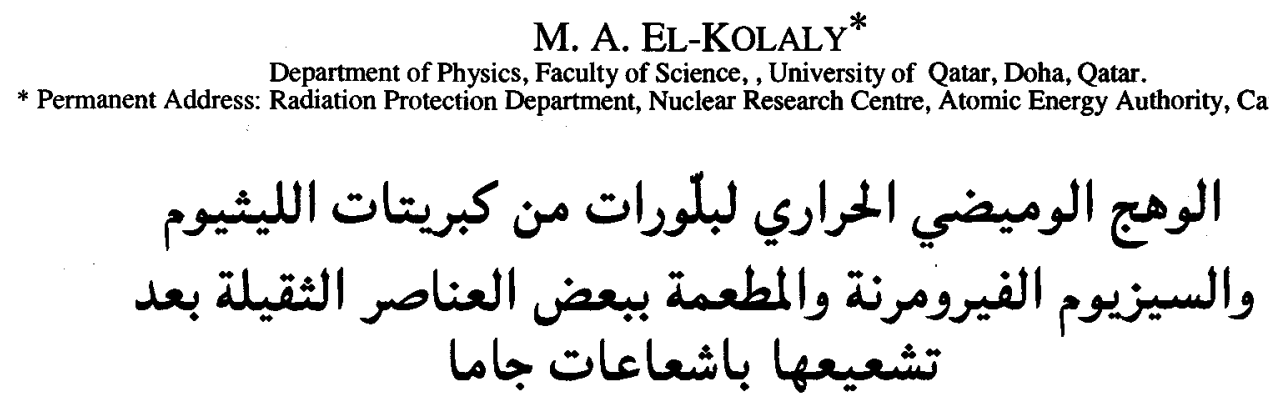

محمــد عبد الفتـاح القللي

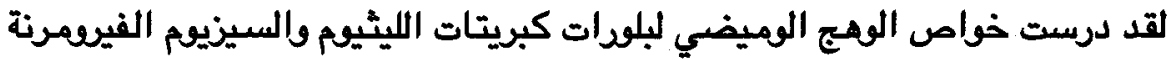

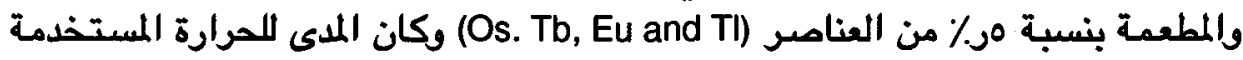

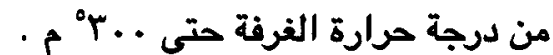

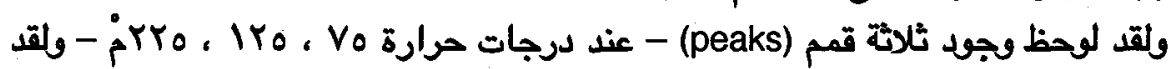

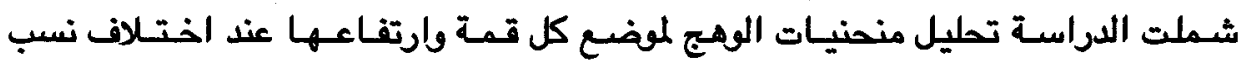

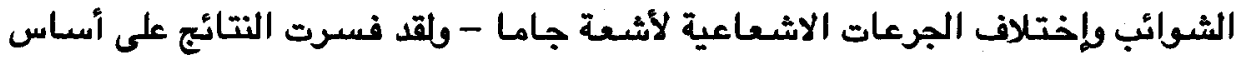

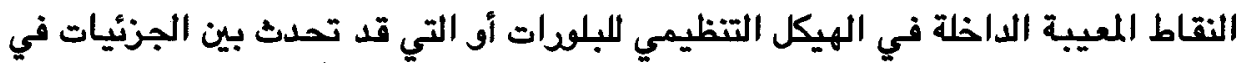

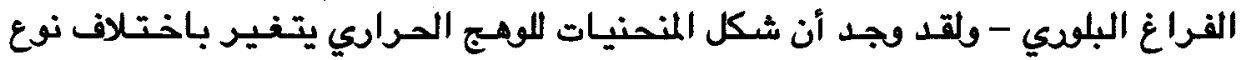

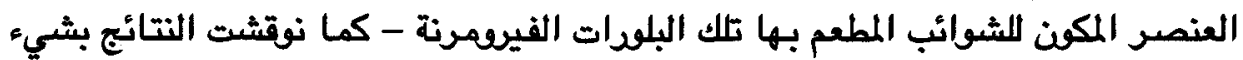

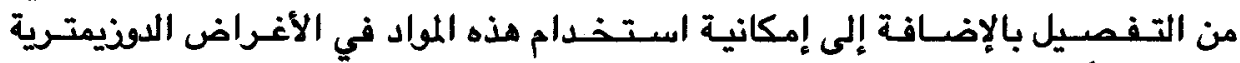

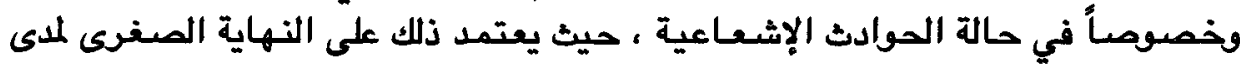

$$
\begin{aligned}
& \text { حساسية تلك المواد للإشعاعات المؤينة . }
\end{aligned}
$$

Key Words: Thermoluminescence, Gamma radiation, Glow curves, Ferroelastic crystal, Dosimetry.

\section{ABSTRACT}

Thermoluminescence (TL) glow curves of lithium cesium sulphate ferroelastic crystals LiCsSO4 doped with $0.5 \%$ of Os, Tb, Eu and Tl have !reen studied in the temperature range from room temperature to $300^{\circ} \mathrm{C}$. Three peaks are observed at about 75,125 and $225^{\circ} \mathrm{C}$. The study include also analysis of peak position and peak height of the glow curves with various dopant ions at different gamma radiation doses. The results are interpreted on the basis of point defects introduced interstitially or substitutionally admixtured into the host lattice. In spite of the host material being the same (LiCsSO4), the glow curve structure changes due to variation of dopant materials ( $\mathrm{Os}, \mathrm{Tb}$, Eu, and $\mathrm{Tl}$ ). The obtained results are discussed in detail. 1 se applicability of such materials for accident dosimetry depends on the lower limit of detection.

\section{INTRODUCTION}

In many cases the properties of imperfect crystals are strongly related to the behaviour of the domain wall in a random medium. This domain wall can co-exist in ferroelastic crystals, because this type of crystals can minimize its free energy by splitting into an optimum number of domains. The energy can be located in the domain wall, and a dense network of domain can provide stress accommodating mechanism $(1,2)$. In a medium with frozen in defects the domain wall deviates from its position and for small concentration of defects the monodomain state is stable against the domain wall formation. The domain wall tends to move if influenced by external or thermal excitations to overcome the barriers. Crystals with general formula MIMIIBX4 in several cases exhibit phase transformation associated with the onset of spontaneous strain in the low and high temperature phase(3-6). This leads in consequence to anomalous changes of their elastic properties $(7,8)$.

The LiCsSO 4 crystals undergo a structural phase transition at about $202 \mathrm{~K}$ from orthorhombic point group $\mathrm{mmm}$ to low 
temperature monoclinic(7). This is strongly confirmed by calorimetric measurements(8), which demonstrated specific heat anomaly at $\mathrm{Tc}$ in the absence of the latent heat and in addition no thermal effect was observed.

Optical, electro- paramagnetic resonance and nuclear magnetic resonance studies of $\mathrm{LiCsSO} 4$ crystals have been reported (9-10). The phosphor were made in the period between 1954 and 1960 from pure sample only(12), and are consequently much less sensitive. Systematic studies of various activators and activator combinations led to the development of sensitive phosphors. From these activators $\mathrm{Os}, \mathrm{Tl}, \mathrm{Eu}$ and $\mathrm{Tb}$ are generally believed to be those of dosimetric importance. The optimum sensitivity results from addition of $0.5 \%$ of the dopant(13-15).

In this paper the thermoluminescence technique was used to find out the effect of gamma radiation induced thermoluminescence in $\mathrm{LiCsSO} 4$ doped with $0.5 \%$ of $\mathrm{Os}, \mathrm{Tl}$, Eu\&Tb.

\section{EXPERIMENTAL}

The materials used in this work were prepared from high purity Lithium sulphate and Cesium sulphate mixed in equimolar quantities and then heated to $900^{\circ} \mathrm{C}$ for 4 hours in a platinum crucible. The obtained $\mathrm{LiCsSO} 4$ matrix was left to cool to room temperature in the oven. The resulting solid was powdered again and divided into five portions. The first four patches were doped with $\mathrm{Tl}, \mathrm{Tb}, \mathrm{Os}$ and Eu separately. The concentration of each dopant was $0.5 \%$ by weight. The fifth part was kept un-doped. Each of the doped samples was mixed thoroughly to form a uniform distribution and then heated at $950^{\circ} \mathrm{C}$ for 1 hour. After cooling the produced solid samples were ground to 80-120 mesh. The prepared samples were then 8 -irradiated with different doses (using Co-60 gamma cell 220 manufactured by Atomic Energy of Canada LTd.). The gamma dose rate was $225 \mathrm{~Gy} / \mathrm{min}$.

The thermoluminescence measurements were carried out using a Harshaw $2000 \mathrm{~A}+\mathrm{B}$ TL reader from room temperature up to $300{ }^{\circ} \mathrm{C}$. The heating rate was $5^{\circ} \mathrm{C} / \mathrm{sec}$. To avoid sample oxidation, the system was flushed with pure and dry N2 gas between and during measurements. For precision, each measurement was made twice.

\section{RESULTS AND DISCUSSION}

Thermoluminescence of $\mathrm{LiCsSO} 4$ has been measured at different gamma radiation exposure doses $(0.056,320$ and 675 $\mathrm{KGy}$ ). The glow curves have been measured in the range from room temperature up to $300{ }^{\circ} \mathrm{C}$. Fig (1) a, b \& c represents typical TL glow curves. At low radiation doses $(\approx 0.056$ KGy) the glow curves show two isolated peaks at 75 and $125^{\circ} \mathrm{C}$ for non-doped samples. Doped ones show change in glow curve structure in both peak height and peak temperature. This behaviour can be attributed as due to the fact that when the ferroelastic $\mathrm{LiCsSO} 4$ crystals are irradiated with gamma radiation the spontaneous strain tensor will change creating an internal stress which tends to diminish the TL responses, the number of populations are decreased by the moderate radiation doses and the $\mathrm{TL}$ glow peak becomes triplet with higher TL intensities. By further irradiation $(\approx$ $675 \mathrm{~K} \mathrm{~Gy}$ ) the TL trap gains high sensitization and gives high $\mathrm{TL}$ response, as is clear from Fig (1-c)

Upon insertion of $0.5 \%$ by weight of $\mathrm{Tl}$, the $\mathrm{TL}$ response shows two overlaping peaks which increase as the dose increases. In case of Eu, the response shows two faint TL glow peaks. At higher doses this becomes a triplet. In constrast Os

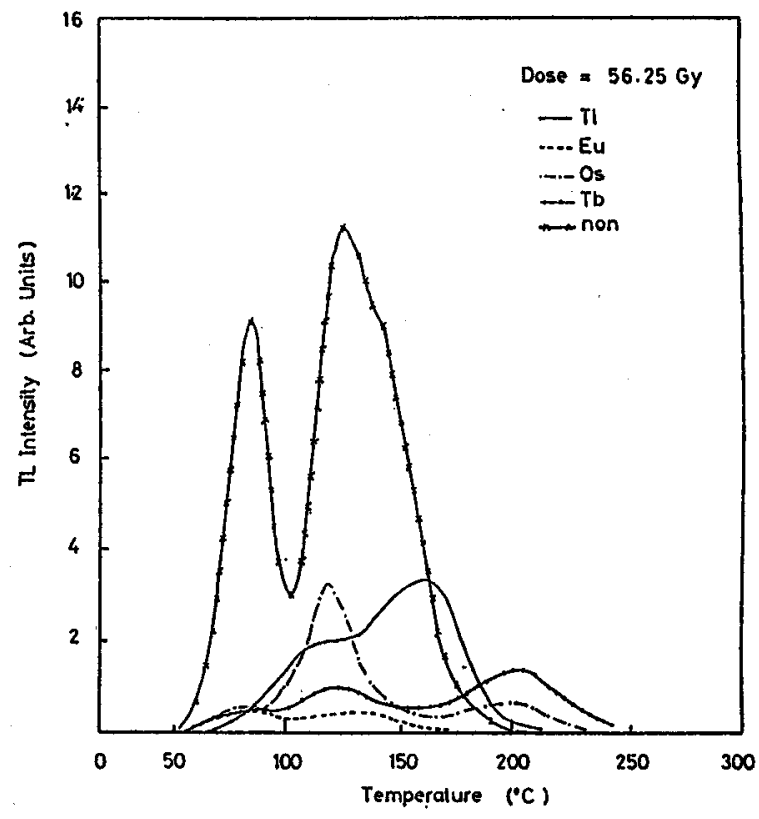

Fig. 1.(a) $\mathrm{TL}$ glow curves of $\gamma$-irradiated $\mathrm{LiCsSO}_{4}$ crystals both non-doped and doped with $0.5 \%$ by weight of Tl-Eu-Os-Tb. $\gamma$-radiation dose $=56.25 \mathrm{~Gy}$.

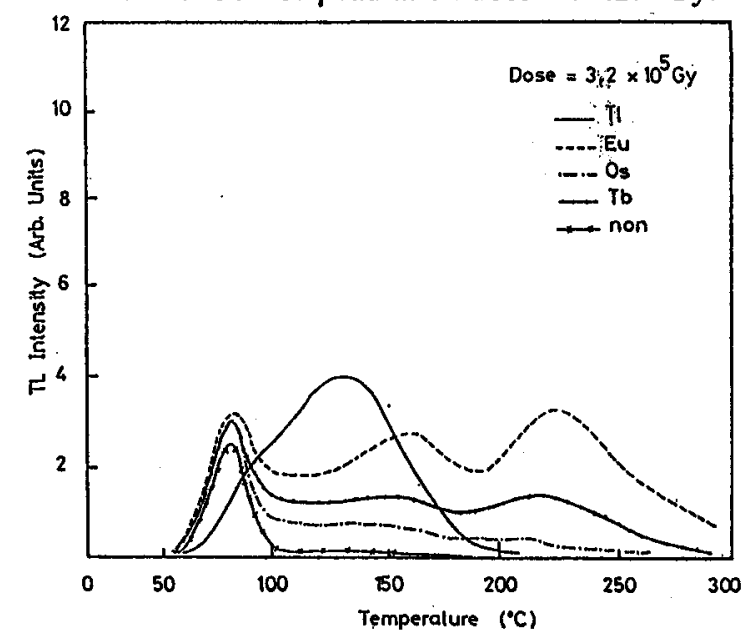

(b) $\mathrm{TL}$ glow curves of $\gamma$-irradiated $\mathrm{LiCsSO}_{4}$ crystals both non-doped and doped with $0.5 \%$ by weight of Tl-Eu-Os-Tb. $-\gamma$-radation dose $=320 \mathrm{kGy}$.

shows a different behaviour in which the TL peak changes slowly with gamma dose till $320 \mathrm{~K}$ Gy which is followed by a sharp increase in the TL response at higher doses. With $\mathrm{Tb}$ the response shows three faint peaks at low doses. The first peak becomes sharp while the other two change slightly at medium radiation level. The three peaks become sharp and isolated with higher radiation doses.

Fig (2-a) shows the TL intensities of the first peak (PKI) at $75^{\circ} \mathrm{C}$ versus radiation doses. It is clear from this figure that the $\mathrm{TL}$ intensities of $\mathrm{LiCsSO} 4$ samples doped with $\mathrm{Tb}$, Os and Eu increase as the radiation doses are increased. In case of non-doped samples this increase goes faster than for doped samples. There are no glow peaks due to $\mathrm{Tl}$ in this range of dose. This behaviour can be explained by the fact that the replacement of $\mathrm{Cs}$ by $\mathrm{Tl}$ breaks the symmetry of the lattice and couples linearly to the order parameter (spontaneous strain tensor). On the other hand $\mathrm{Os}, \mathrm{Tb}$ and Eu can replace Li or $\mathrm{Cs}$ and the influence of defect on TL glow curve depends strongly on the relaxation of the defected cells which tend to order themselves in a way to favour orientation of the order parameter. 


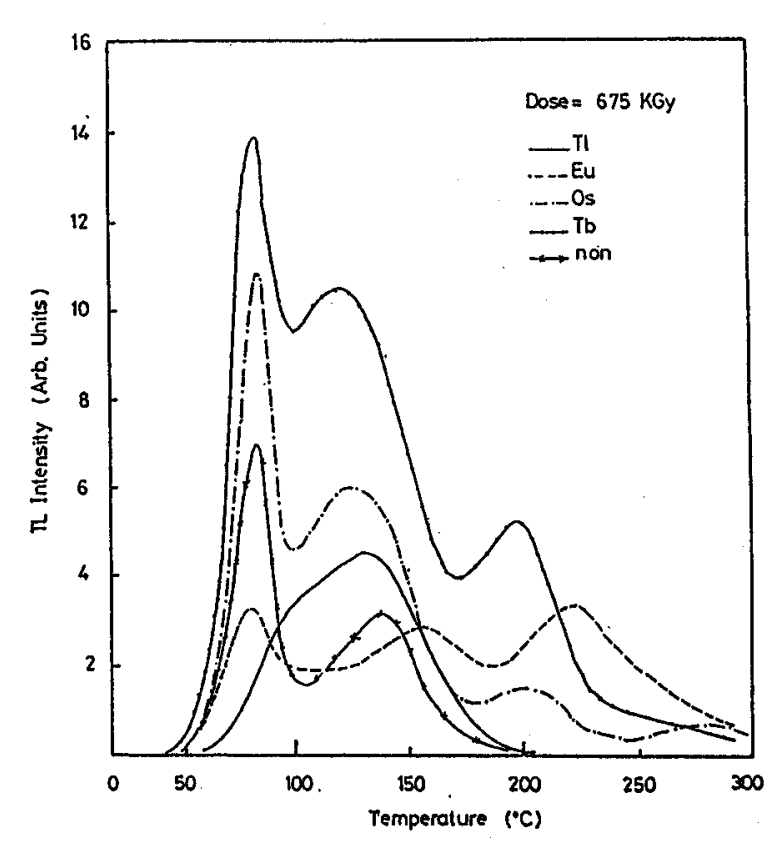

(c) TL glow curves of $\gamma$-irradiated $\mathrm{LiCsSO}_{4}$ crystals both non-doped and doped with $0.5 \%$ by weight of $\mathrm{Tl}$ - Eu - Os - Tb. $\gamma$-radiation dose $=675 \mathrm{~K} \mathrm{~Gy}$.

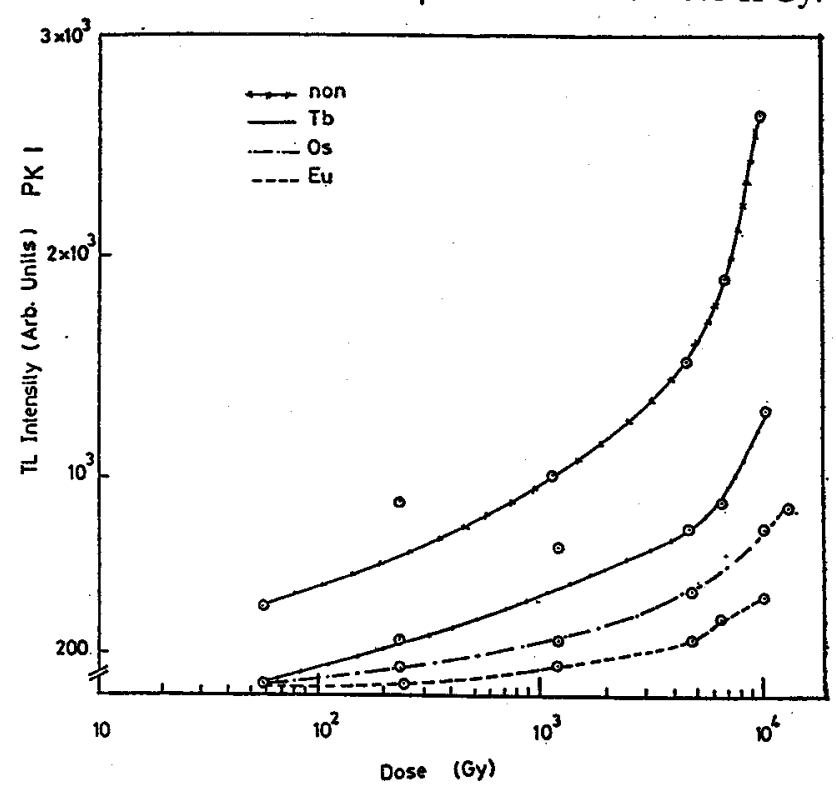

Fig. 2 (a) TL response of $\mathrm{LiCsSO}_{4}$ samples both non-doped and doped with $0.5 \%$ of $\mathrm{Tb}-\mathrm{Os}-\mathrm{Eu}$ versus gradiation dose for the first peak (PK I).

Fig (2-b) shows also the TL peak intensities (PK II) at $\left(125^{\circ} \mathrm{C}\right)$ as a function of radiation doses. It is clear from this figure that non-doped samples as well as Tl, Tb and Os doped samples show an increase with dose. In case of $\mathrm{Tl}$ doped samples the increase in TL glow peaks goes faster approximately in the same way as in the non-doped samples. This can be also attributed to the fact that $\mathrm{Tl}$ in the LiCsSO4 sample replaces $\mathrm{Cs}$ in the lattice. This leads to two phase systems and so, it behaves differently. However, in the other cases $\mathrm{Tb}$, Os and $\mathrm{Eu}$ are sited in the interstitial position and these behave similarly.

Fig (3-a) shows variation of peak temperature (The first peak I at $75^{\circ} \mathrm{C}$ ) with radiation dose. It is clear from this figure that peak temperature decreases as radiation dose increases for non-doped samples as well as for Os and Eu doped LiCsSO4

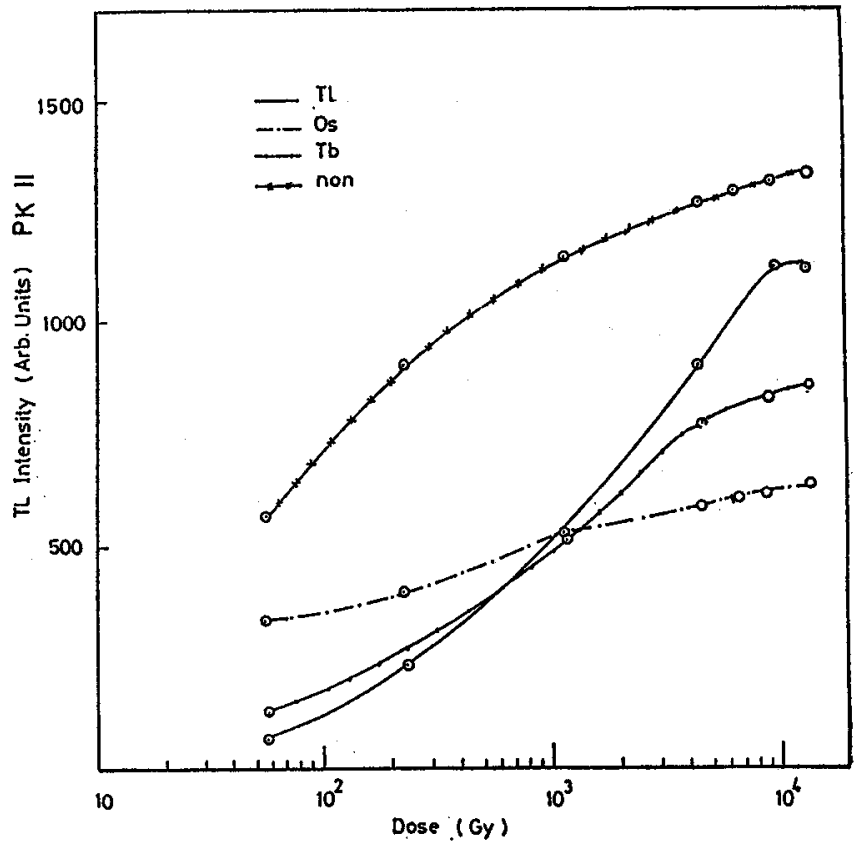

(b) TL response of $\mathrm{LiCsSO}_{4}$ samples both non-doped and doped with $0.5 \%$ of $\mathrm{Tl}$, Os, Tb versus gradiation doses for the second peak (PK II).

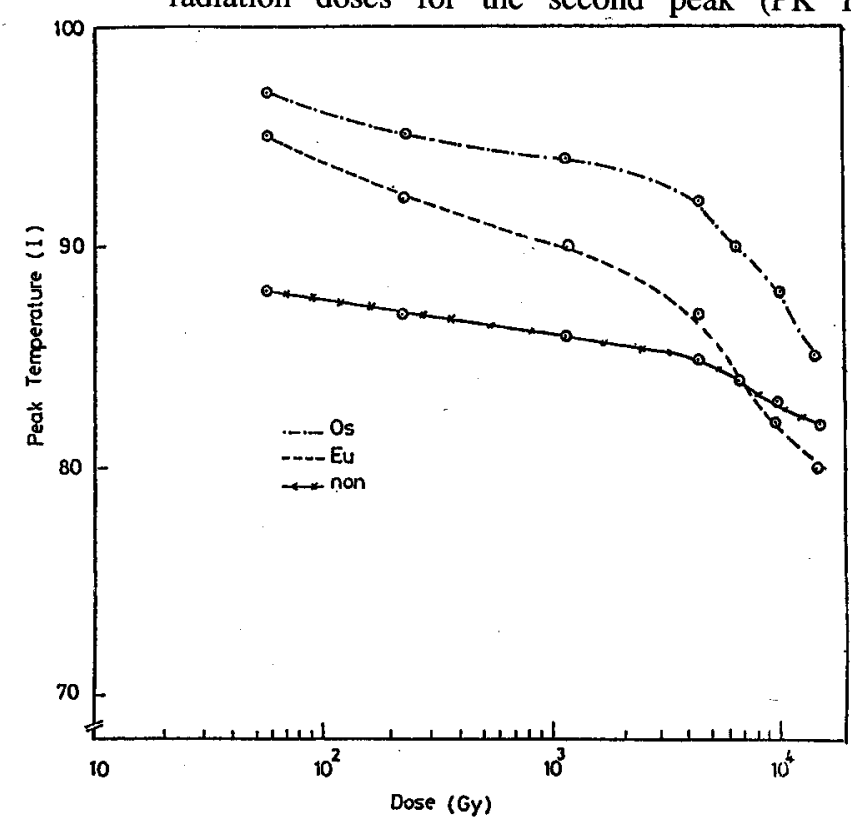

Fig.3. (a) Variation of the TL peak temperature (peak I) of $\mathrm{LiCsSO}_{4}$ samples doped with Os and Eu versus radiation gamma doses.

samples. On the other hand, Fig (3-b) shows the variation of peak temperature (Peak II at $150^{\circ} \mathrm{C}$ ) with radiation dose for pure (non-doped) as well as $\mathrm{Tl}, \mathrm{Os}$ and $\mathrm{Tb}$ doped samples. The peak temperature increases slightly with radiation dose. On the contrary, in case of $\mathrm{Tl}$ doped samples, the peak temperature decreases with gamma radiation dose. This decrease in the TL intensity may be attributed to the scattering mechanism of the defect with spontaneous strain. Upon increasing radiation doses, the spontaneous strain diminishes, and therefore this defect is dominated.

\section{CONCLUSIONS}

From the obtained results one can conclude that heavy metal impurities (Tl, Tb, Os and Eu) play an important role in the thermoluminescence of $\mathrm{LiCsSO}_{4}$ ferroelastic crystals. 


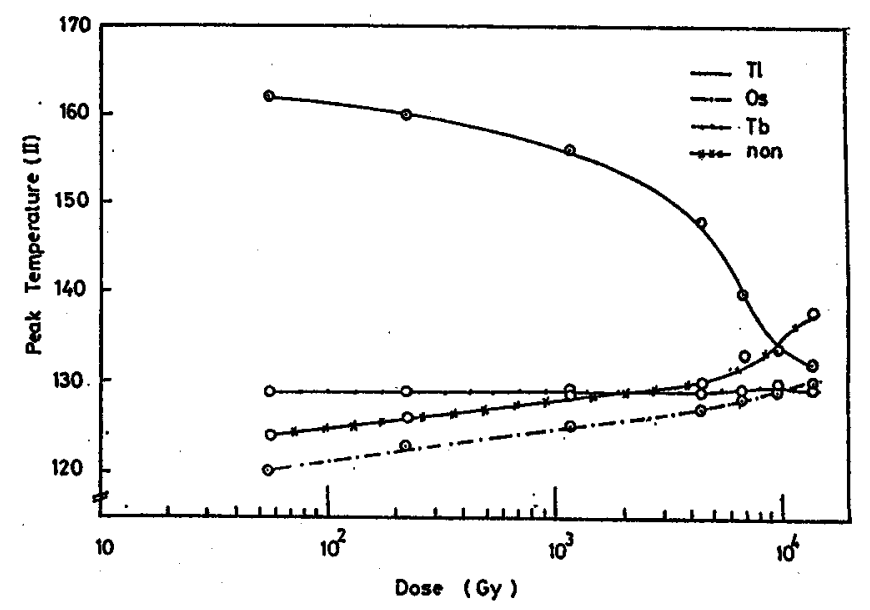

(b) Variation of the TL peak temperature (peak II) of $\mathrm{LiCsSO}_{4}$ samples doped with $\mathrm{Tl}, \mathrm{Os}, \mathrm{Tb}$ versus $\mathrm{g}$ radiation doses.

Also, adding $0.5 \%$ of $\mathrm{Tl}$ by weight to the host lattice gives high TL sensitivity as indicated by the second peak around $150^{\circ} \mathrm{C}$ up to $1 \mathrm{~K}$ Gy gamma radiation dose. Therefore, using these materials in the field of radiation dosimetry is useful in this dose range.

\section{ACKNOWLEDGMENT}

The author is thankful to Prof. Dr. M. E. Kassem for keen interest and useful discussion. Thanks also to Prof. Dr. L. I. Al-Houty, Head of the Physics Department, Faculty of Science, University of Qatar, for her continuous encouragement.

\section{REFERENCES}

[1] Malis, T. and H. Gleiter, 1979. On the structure of ferroelectric - paraelectric transformation interface in barium titanate. I. Basic structure and characteristics. J. Appl. Phys. 50 (7): 4920-4923.

[2] Metrat, G., 1980. Theoretical determination of domain structure at transitions from twinned phase: Application to the tetragonal-orthorhombic transition of $\mathrm{KNbO}_{3}$. Ferroelectrics, 26 (1-4): 801-804.

[3] Tuszynski, J.A., B. Mroz, H. Kiefte and M.J. Clouter, 1988. Comments on the hysteresis loop in Ferroelectric $\mathrm{LiCsSO}_{4}$. Ferroelectrics, 77: 111-120.

[4] Pakuluski, G., T. Breczewski, B. Mroz and T. Krajewski, 1987. Temperature dependence of ferrobielectric properties of lithium calsium sulphate. Ferroelectrics, 74 (3-4): 375-380.

[5] Kassem M.E., 1992. Ferroelectric-assisted phase transition to fast ionic conduction in $\mathrm{LiKs}_{4}$-A correlative AC conductivity specific heat study. Materials Lett. 15 (3): 162-166.

[6] Krajewski, T., T. Breczewski, M. Kassem and B. Mroz, 1983. Ferroelasticity and internal friction in $\mathrm{LiKSO}_{4}$ crystals. Ferroelectrics, 55 (1-4): 811-814.

[7] Ozeki, H. and A. Sawada, 1982. Acoustic softening in ferroelastic $\mathrm{LiCsSO}_{4}$ crystal J. Phys. Soc. Japan. 51 (7): 2047-2048.

[8] Kruglik, A.I., M.A. Simonov, E.P. Zhelezin and N.V. Belov, 1979. Crystal structure of phases I and III of cesium lithium sulphate. Doklady Akad. NAuk SSSR. 247, (4-6): 1384-1387.

[9] Alexsandrov, K.S., L.I. Zherebtsova and I.M. Iskornev, A.I. Kruglik, O.V. Rozanov and I.N. Flerov, 1980. Investigation of structural and physical properties of calsium lethium double sulphate. Fizika Tverdogo Tela. 22 (12): 3673-7.

[10] Zamkov, A.V. and A.T. Anistratov, 1982. Piezooptic investigation of the ferroelastic phase transition in $\mathrm{CsLiSO}_{4}$. Fizika Tverdogo Tela. 24 (5): 1524-6.

[11] Morais, P.C., G.M. Bibeiro and A.S. Chaves, 1984. ESR study of the ferro-elastic phase transition in $\mathrm{CsLiSO}_{4}$. Solid State Communications, 52 (3): 291-2.

[12] Holuj, F., 1986. NMR of $\mathrm{Cs}^{133}$ in $\mathrm{LiCsSO}_{4}$. Ferroelectrics. 67 (2-4): 103-107.

[13] Mroz, B., H. Kiefte, M.J. Clouter and J.A. Tuszynski, 1987. Brillouin scattering studies of the ferroelastic phase transition in $\mathrm{LiCsSO}_{4}$. Phys. Re. B. 36 (7): 3745-3754.

[14] El-Kolaly, M.A., M.E. Kassem, A.A. Higaz, L.Z. Ismail and L.I. Al-Houty, 1992. Effect of gamma irradiation on thermo-luminescence and thermo-dynamic parameters of $\mathrm{LiKSO}_{4}$ doped with $\mathrm{Nd}_{2} \mathrm{O}_{3}$. Radiation Effects and Defects in solids, 124 (4): 437-442.

[15] Kassem, M.E., M.A. El-Kolaly and K.Z. Ismail, 1993. Thermal and thermoluminescence properties of gamma-irradiated $\mathrm{LiNaSO}_{4}$ Crystals doped with $\mathrm{Tl}$. Materials Lett. 16 (2-3): 102-107. 